\title{
Redes de Cooperación Científico-Tecnológicas en Contextos Intersectoriales
}

\author{
Freddy Marín-González(1), Rosalba Talavera(2), Alicia Inciarte-González ${ }^{(1)}$ e Inírida Avendaño-Villa(1) \\ (1) Departamento de Humanidades, Universidad de la Costa, Atlántico - Colombia \\ (e-mail: fmarin1@cuc.edu.co; ainciart1@cuc.edu.co; iavendano@cuc.edu.co) \\ (2) Departamento de Ciencias Formales, Universidad del Zulia, Falcón - Venezuela \\ (e-mail: talavera_p@hotmail.com)
}

Recibido Jul. 9, 2018; Aceptado Sep. 24, 2018; Versión final Dic. 6, 2018, Publicado Jun. 2019

\begin{abstract}
Resumen
Este artículo se orienta a la conformación de una red de cooperación científico-tecnológica en el ámbito interorganizacional e intersectorial, desde la gestión participativa de actores locales. Al respecto, se concibe una interfaz relacional caracterizada por el intercambio y transacción de conocimiento. Se trabaja con métodos de razonamiento lógico que derivan en la inferencia y análisis propositivo desde los aportes de la teoría de redes, cooperación y grafos. En el componente empírico se abordan actores clave del sector universitario gobierno-empresa, localizados en el estado Falcón, Venezuela. Los resultados muestran que la dinámica de la gestión institucional viabiliza la configuración de la red con la definición de su estructura y funcionalidad. Se concluye sobre la importancia de la gestión participativa para la integración de redes científico-tecnológicas, mediante flujos de cooperación entre los equipos de trabajo que constituyen nodos de la red.
\end{abstract}

Palabras clave: redes científico-tecnológicas; gestión participativa local; cooperación; intersectorialidad; intercambio de conocimiento

\section{Scientific-Technological Cooperation Networks in Intersectoral Contexts}

\begin{abstract}
This article is oriented to the creation of a scientific-technological cooperation network in the interorganizational and intersectoral field, from the participative management of local actors. In this regard, a relational interface characterized by the exchange and transaction of knowledge is conceived. The work is done using logical reasoning methods that derive in the inference and proactive analysis from the contributions of the theory of networks, cooperation and graphs. The empirical component addresses key actors in the universitygovernment-business sector, located in the state of Falcón, Venezuela. The results show that the dynamics of the institutional management enables the configuration of the network with the definition of its structure and functionality. The conclusion highlights the importance of the participative management for the integration of scientific-technological networks, by means of flows of cooperation between the work teams that constitute the nodes of the network.
\end{abstract}

Keywords: scientific-technological networks; local participatory management; cooperation; intersectoriality; knowledge exchange 


\section{INTRODUCCIÓN}

La configuración de redes científico-tecnológicas requiere de la gestión participativa de actores locales en diferentes subsistemas sociales que comparten un mismo territorio; en este sentido lo territorial trasciende la concepción de ámbito físico - geográfico para ubicarse en el plano de las interacciones y flujos relacionales entre actores, entidades y organizaciones. Por tanto, en el territorio se configuran tejidos desde la convergencia de perfiles, funciones, tareas y comportamientos, permite la articulación de nodos o espacios de integración a través de mecanismos de comunicación, sinergia y consenso. La funcionalidad y ubicuidad de la red determinan su impacto, alcance y utilidad, asociado a la generación de recursos, relación costo beneficio y mejoramiento de la calidad de vida. Las redes representan en sí mismas una estrategia de las regiones para impulsar procesos de desarrollo sostenible.

La conformación de estas redes supone la generación de capacidades en el ámbito local y regional, asociadas a la disposición y uso de las tecnologías de información. Desde la perspectiva de García et al., (2017), es necesario proveer opciones tecnológicas, que en un plano operativo catalicen acciones cooperativas entre entidades individuales y organizacionales (socios y empresas) mediante flujos relacionales de conocimiento con base en procesos de transacción e intercambio. Al respecto se puntualiza que, en contextos donde actualmente se generan brechas importantes para alcanzar el desarrollo humano y social, resulta imperativo la definición de estrategias que permitan a las organizaciones generar capacidades, expresadas en habilidades, recursos, rutinas, flujos de conocimiento, como base para fortalecer su ciclo de vida en los estadios de crecimiento y desarrollo (Lovera et al., 2008, Simanca et al., 2016). La concepción de estrategias orientadas al propósito mencionado, se dinamizan en el ámbito intra e interorganizacional, así como también en espacios de intersectorialidad; es allí donde la configuración de redes, de diferente orden, incluidas las de carácter científico - tecnológica contribuyen a la consecución de metas y objetivos comunes. López, Marulanda y López (2016), destacan de las redes intersectoriales, su pertinencia como estrategia para desarrollar capacidades asociadas al trabajo en equipo, mediante la participación de los actores en procesos de cooperación que permitan responder de forma ágil y efectiva a los desafíos de un mundo globalizado y en contante transformación. En este sentido, Espinoza (2011), señala que la producción de conocimiento orientado y aplicado, dirigido hacia la innovación, es el centro de interés que lleva a las organizaciones a vincularse y luego a integrarse en relaciones de cooperación. Emerge la intersectorialidad como ámbito de consenso para establecer alianzas de conocimiento, respaldadas en las capacidades y fortalezas, que aportan las entidades individuales y organizacionales para llegar a un fin común.

A decir de Osca-Lluch y González-Sala (2017), los procesos de cooperación científica varían según la naturaleza de las organizaciones y sectores relacionados, así como también las disciplinas científicas, que determinan las características del conocimiento producido, transferido y compartido; por ejemplo, en el ámbito académico, desde la perspectiva de Cardozo-Montilla (2012), resulta imperativo adoptar la noción de trabajo de investigación en red como un mecanismo capaz de liberar la tensión asociada a la necesidad de la universidad de transformarse en una organización innovadora en correspondencia con su misión formadora. Uno de los condicionantes que incide para establecer redes de cooperación, está representado por la propia realidad y sus problemas; en contextos complejos se requieren abordajes interdisciplinarios para fortalecer la creación de equipos; ello supone, la participación activa de los actores en la búsqueda de la integración intersectorial en el ámbito local, regional, nacional e internacional. Autores como Obando y Delgado (2007), De Rolt et al. (2017) destacan las relaciones de cooperación en el ámbito científico - tecnológico, como un proceso clave para la madurez del sistema, fundamentado en la generación de capacidades de los actores locales, con su perfil como agentes de innovación; en los referidos contextos complejos, las oportunidades de crecimiento y desarrollo se maximizan en grupos, asociaciones y comunidades fuertemente unidos e inspirados por el deseo de lograr metas comunes. Con este argumento coincide Marín et al., (2017), quienes asumen la gestión participativa de los sistemas humanos, desde su carácter evolutivo y donde los actores fortalecen competencias para adaptarse a entornos con alto grado de turbulencia e impredectibilidad.

Este artículo de intencionalidad propositiva se orienta a analizar las condiciones que permiten la conformación de una red de investigación científica - tecnológica en el ámbito interorganizacional e intersectorial, desde la gestión participativa de actores locales. Por ello se hace necesario conceptualizar una interfaz relacional caracterizada por el intercambio y transacción de conocimiento entre unidades de investigación, gobierno local y otras instituciones del sistema social. Se derivan categorías de análisis desde los aportes de las teorías de redes, cooperación y grafos como principales referentes de entrada. Así mismo, se aborda un componente empírico orientado a la configuración de una red científico - tecnológica, que desde su gestión y dinámica funcional pretende contribuir a procesos de participación y desarrollo local; para efectos del estudio se trabaja con organizaciones representativas de los sectores: universitario - gobierno - empresarial, localizados en el estado Falcón - Venezuela, con la intención de que la propia dinámica de estos espacios de gestión viabilice la configuración del mencionado tejido relacional, y la definición de su estructura y funcionalidad pueda servir de modelo para su aplicación en otros contextos de características similares. 


\section{FUNDAMENTACIÓN TEÓRICA - CONCEPTUAL}

El funcionamiento de las redes de cooperación como expresión de las relaciones interorganizacionales, puede ser comprendido desde la multiplicidad de vínculos que definen flujos de conocimiento entre los diferentes sectores al establecer alianzas estratégicas para la participación en procesos de crecimiento y desarrollo social. Marín et al., (2014), destacan que las redes científico - tecnológicas se configuran en tres planos de integración: a nivel macro se ubican redes intersectoriales, que se fundamentan en espacios cognitivos intersistemas e intersectores; en lo meso relacional, el conocimiento se disemina en lo organizacional con flujos externos e internos, creándose una dinámica convergente; mientras que el plano micro está asociado al perfil de los actores y sus formas de abstracción y representación de la realidad.

La forma de establecer nexos directos e indirectos entre los actores se explica con base en la teoría de grafos; para Wasserman y Faust (1994), constituyen representaciones abstractas de orden lógico - formal que contribuyen a la concepción relacional - sistémica, con énfasis en la disposición gráfica (componente anatómico - estructural), conectividad, densidad, medidas de grado de centralidad. Cárdenas et al., (2015), resaltan que la teoría de grafos como un dominio de las matemáticas discretas, permite aproximarse a una valoración cualitativa y cuantitativa de la dinámica estructural - funcional de la red. Representa un campo de conocimiento para derivar conceptos y métodos que coadyuvan a la comprensión de las propiedades estructurales y sus formas de cuantificarse; orienta la operatividad del modelo de red, al representar los individuos (nodos) y sus vínculos (aristas) mediante un grafo; consecuentemente se infiere que los grafos permiten el conocimiento de los componentes estructurales de la red.

En un sentido más amplio, el perfil operativo de una red expresa flujos de comunicación, intercambio y transacción entre actores; normas y valores que condicionan conductas y formas de comportamiento. En la trama relacional se evidencian posiciones funcionalmente diferenciables, asociadas no solo a las entidades individuales que se relacionan, sino también al desempeño de la red en su conjunto. Aun cuando se crea una identidad compartida, siempre hay un rol operativamente relevante de cada posición (tipo de actor), trabajando en temas específicos, mediante la compartición de información, conocimiento, infraestructura, costos, recursos; se pretende fortalecer la cooperación en áreas de desarrollo estratégico en el ámbito científico tecnológico.

La conformación de redes, puede ser analizada con base en sus propiedades estructurales; se mencionan, la densidad que valora la cantidad promedio de lazos, así como la existencia y funcionamiento de los grupos de interés y sus compromisos formales, esto determina los grados de conectividad; igualmente, se considera la cercanía, capacidad de un actor para alcanzar e influenciar a todos los nodos de la red; mientras que la centralidad, atiende a los nodos más importantes, como espacio de confluencia de múltiples flujos, permite identificar los actores que ejercen un mayor poder/mayor motricidad sobre la totalidad del sistema. Desde los aportes de Borgatti (2005), Bakkalbasi y Krichel (2006), Bozdogan (2013), Robinson et al., (2013), Li et al., (2016), la motricidad del sistema está asociada a la posición ventajosa, según grado de centralidad, que tiene un actor sobre los demás; implica una mayor influencia o conexión con el resto de actores; permite identificar los nodos más determinantes en función de su impacto que forman parte de la red.

Por su parte, Villanueva-Felez, Fernández-Zubieta y Palomares-Montero (2014), retoman las ideas expuestas por Uzzi (1997), para destacar que en el análisis de redes, además de atender el componente estructural, se debe observar la dinámica funcional - relacional, en una dimensión personal (actores) y otra organizacional. Los principios de integración, convergencia, complementariedad e integración entre ambas dimensiones preconfiguran formas de comportamiento; de allí que, el perfil individual de los actores incide de forma significativa en las cualidades de la producción científica - técnica; por su parte, la dinámica de la red como totalidad institucional afecta su carácter cuantitativo.

Fundamentado en las vías de conectividad para impulsar el acercamiento entre actores, el análisis de redes supone la conceptualización de una interfaz, como espacio de interacción donde se representan los flujos que en múltiples direcciones y sentidos, permiten el acercamiento y vías de conectividad entre los nodos centrales y periféricos. La dinámica descrita por la interfaz relacional se basa en un sentido de transacción e intercambio de conocimiento, con fundamento en la alianza como principal expresión de los procesos de cooperación desde sus principios de complementariedad, convergencia e integración; en la interfaz se propicia la compartición de conocimiento de base interdisciplinaria, respaldado en la generación de capacidades que en lo personal y organizacional desarrollan las entidades interactuantes.

El sentido de transacción e intercambio como base de las alianzas de conocimiento, implica flujos de interacción en múltiples direcciones; ello supone la definición de estrategias que se articulen en atención a: i) Asociación de organizaciones que persigan un objetivo común, resolviendo problemas que requieran soluciones innovadoras e inmediatas; ii) Fortalecer el tejido de relaciones sólidas, con vínculos sostenibles 
en el tiempo, y donde se involucren actores, procesos, inputs y outputs, contextos, sistemas; buscando la concordancia entre los procesos internos y el entorno; iii) Propiciar el establecimiento y seguimiento de convenios con organismos locales y nacionales que faciliten u ofrezcan beneficios e intercambios a investigadores; iv) Generar respuestas con un alto nivel de compromiso y confianza, a fin de garantizar el cumplimiento de las tareas inherentes a la resolución de problemas; v) Enfocar la capacitación profesional desde la integración entre actores, generando beneficios mutuos a través de la producción de bienes, prestación de servicios, consultorías y plan de estímulos a la labor investigativa; vi) Disponer canales formales e informales de comunicación efectiva y oportuna entre socios, con la intención de fortalecer los acuerdos, acciones y tareas para la consecución de objetivos; vii) Definir los nodos más significativos de la red desde la convergencia, integración e impactos de flujos, para actuar como apoyo coordinado, concertado y focalizado; viii) Fortalecer una cultura de trabajo con los actores centrada en flujos de conocimiento destinado a detectar las demandas del entorno; ix) Disponer una estructura de sustento para el fortalecimiento de relaciones de vinculación, asociación y cooperación científico - técnica.

En el ámbito de las relaciones interorganizacionales la configuración de redes, constituye en sí misma una estrategia de significativa pertinencia, para la integración de los actores en la consecución de objetivos, que de forma independiente no sería posible alcanzarlos. Para Gutiérrez (2017), la concepción de red fundamentada en la definición de alianza estratégica, implica formas institucionales de gobernanza de las relaciones estratificadas de cooperación, con base en el intercambio de conocimiento científico - técnico; se asumen relaciones formales entre actores de los diferentes sectores sociales intervinientes. En este sentido, Sacchetti y Tortia (2016), destacan dos tipos polarizados de redes, por un lado, se expresan formas heterogéneas de coordinación basadas en la cooperación y la ayuda mutua, y por el otro, puede operacionalizarse a través de una coordinación jerárquica basada en la dirección exclusiva. Al respecto, Huang et al., (2018), sostienen que es necesario proponer estrategias de aprendizaje de los actores, de manera tal que, se promuevan formas de comportamiento basadas en la cooperación. Consecuentemente, la dinámica de interacción entre organizaciones y sectores sociales, lleva a plantearse la necesidad de configurar sistemas de integración y complementariedad interorganizacional, como clave para fortalecer los flujos de conocimiento entre los diferentes nodos de la red; por tanto, es necesario participar en procesos de innovación, que potencien la competitividad desde la generación de capacidades en los actores, para participar en investigaciones cuyo impacto se valide en el radio intersectorial de las redes conformadas.

\section{METODOLOGÍA}

El componente metodológico de la investigación deviene de su contextualización epistemológica en un enfoque lógico racional, centrado en la reflexión y análisis crítico de la realidad estudiada. Autores como Marín et al., (2018), conciben la producción y validación del conocimiento, mediante procesos de abstracción para la generación de un cuerpo teórico; la investigación se fundamentó en constructos referentes como: redes científico - tecnológicas, cooperación, gestión participativa local, dinámica relacional entre actores; estas categorías son sometidas a un tratamiento de deconstrucción para identificar las principales unidades de información y conocimiento. Igualmente, se orienta un diseño propositivo y de análisis de las condiciones para el establecimiento de la red, en sus dimensiones estructurales, funcionales y de contexto; se toma como base un componente empírico, cuya evidencia es obtenida a través de la aplicación de un cuestionario estructurado a actores clave del sector universitario - sector gobierno - sector empresarial, en funciones gerenciales, académicas y técnicas.

El componente empírico permite identificar los sectores que intervienen en la conformación de la red, ubicados en el estado Falcón - Venezuela, específicamente en la Península de Paraguaná; se discriminan de la siguiente forma: 1) Sector universitario: Unidad de Investigación: Laboratorio de Investigación y Servicios Ambientales Universidad del Zulia- Núcleo Punto Fijo (LISA - LUZ NPF), Unidad de Investigación: Universidad Nacional Experimental Francisco de Miranda (UNEFM), Unidad de Investigación Universidad Nacional Experimental de las Fuerzas Armadas (UNEFA), Unidad de Investigación Universidad Nacional Abierta (UNA), Unidad de Investigación Universidad Bolivariana de Venezuela (UBV); 2) Sector Gobierno: Gobernación del Estado Falcón, Alcaldía del Municipio Carirubana, Fundación para el Desarrollo de la Ciencia y Tecnología (FUNDACITE); 3) Sector Empresarial: Petróleos de Venezuela, Complejo Refinador Paraguaná (PDVSACRP), Cámara de Comercio y Cámara de la industria.

El propósito investigativo orienta la conformación de una red local de cooperación científico - tecnológica, de sistema abierto; define una relación estrecha entre el accionar de los actores y la toma de decisiones, quienes se organizan desde su visión estratégica - situacional, para identificar necesidades y potenciar recursos. En este sentido la configuración de la red atiende a la sistematización procedimental identificada en la tabla 1. 
Tabla 1: Etapas del diseño - sistematización procedimental

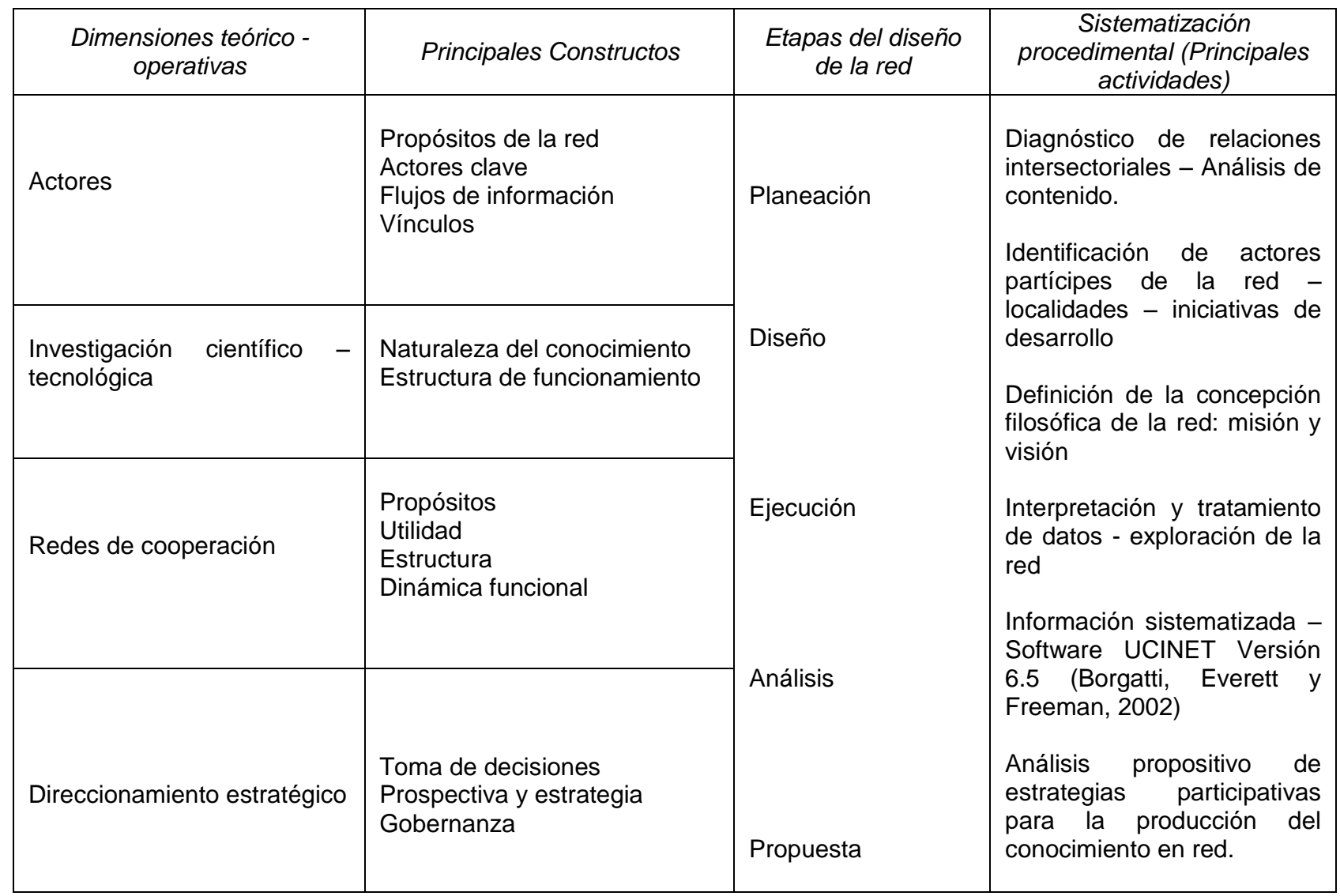

\section{RESULTADOS Y DISCUSIÓN}

La sistematización procedimental que fundamenta las etapas del diseño conduce a organizar los hallazgos en los siguientes apartados: Diagnóstico de las relaciones intersectoriales, Discriminación de actores según perfil de competencias, Concepción filosófica de la red: visión y misión, Exploración de la red con el software UCINET versión 6.5, Análisis propositivo de estrategias participativas en la producción del conocimiento en red, Formación de los integrantes de la red (estrategia de entrada), Capacitación profesional de los miembros integrantes de la red (estrategia de desarrollo), y Talleres de fortalecimiento de los resultados en red (estrategia de cierre)

\section{Diagnóstico de las relaciones intersectoriales}

Del análisis realizado se infiere la necesidad de fortalecer en las universidades, la producción de conocimiento que disponga de cualidades orientadas a generar innovación tecnológica; igualmente, se demanda de la organización empresarial una clara identificación de sus requerimientos en este sentido, y de las condiciones que disponen para que el conocimiento transferido pueda ser incorporado a los procesos productivos que desarrollan en forma de tecnología, adquiriendo su carácter de capitalización.

Al respecto se puntualiza que las universidades, desde sus funciones básicas se acercan al sector empresarial, cuando disponen sus capacidades para participar en la producción de conocimiento susceptible de capitalizarse, vía innovación tecnológica en el ámbito productivo. Etzkowitz (2003), asocia la innovación como una cualidad intrínseca a la complejidad de las relaciones universidad - empresa - gobierno desde la interacción entre sus actores e instituciones. Se generan capacidades para la búsqueda compartida de recursos complementarios para resolver prioridades de desarrollo. Las relaciones interorganizacionales e intersectoriales, están asociados a nuevos engranajes universidad - empresa- gobierno, desde la reinterpretación y revalorización de significados de las formas de relación, como la vía más idónea para alcanzar la sustentabilidad en el ciclo de vida de las organizaciones. Para Cunill-Grau (2014), la noción de intersectorialidad, remite a la integración entre diversos sectores para la solución de problemas sociales complejos desde su multicausalidad, implica relaciones no jerárquicas de cooperación y colaboración.

En este sentido, las demandas emergentes hacia las organizaciones encargadas de la producción y transferencia de conocimiento, conlleva a sus actores a interiorizar la importancia y pertinencia de configurar un modelo de red científico tecnológico, que atienda a una lógica estructura - funcional, a través de la 
observación y descripción de sus condiciones para capitalizar el conocimiento, en el espacio de intersectorialidad sector universitario - sector empresarial. Autores como Vega y Rojo (2010), destacan que las capacidades observables en una relación entre sectores conformados por redes, es de tal impacto, que permite a las organizaciones afrontar la progresividad en los cambios, en virtud del sentido de integración y complementariedad entre los subsistemas interactuantes. Por su parte Azua y Andersen (2010), proponen que la conformación de una red se fundamente en el dominio de estrategias, procesos, tecnología y competencias asociadas al capital humano. Del lado del sector universitario se perciben actividades investigativas cuya tendencia es a incrementar la visibilidad institucional con énfasis en la difusión científica; sin embargo, no se consideran las posibilidades que el conocimiento en sí mismo y la organización como tal, pueden disponer para participar de la innovación tecnológica. Por su parte, desde el sector empresarial el diagnóstico apunta a la necesidad de crear una estructura de sustento, con unidades, procesos y recursos que viabilicen la aplicación del conocimiento transferido vía innovación tecnológica. La efectividad en la concreción de mecanismos relacionales de cooperación y autoaprendizaje entre organizaciones del sistema social trae como resultado el funcionamiento por estancos, lo cual inhibe el sentido de totalidad intrínseca a este tipo de sistemas.

Desde los aportes de Power (1990); Smith y Van de Ven (1994); Dyer y Singh (1998); Sebastián (2000); Gutiérrez et al., (2006); Obando y Delgado (2007); Dushnitsky y Myles; (2009); Weber, Bauke y Raibulet (2016); Weber et al., (2017) se asume la concepción de aprendizaje interorganizacional, como fundamento de las relaciones de cooperación y gestión en la conformación de redes. Se establecen rutinas para compartir conocimiento, con base en la obtención de información clave, que permita adaptarse a entornos cambiantes y complejos. Este proceso implica el establecimiento de acuerdos formales o informales, con sentido de transacción e intercambio. Refiere la búsqueda de objetivos comunes, mediante la definición de un plan de acción fundamentado en el compromiso activo de los involucrados. Una gestión participativa en la producción del conocimiento requiere un alto sentido de reflexión acerca del contexto donde se produce, con la finalidad de asumir posiciones interpretativas de valor que sean resultado del análisis, comprensión de la realidad, hipótesis verificables y diseño de propuestas. Es importante precisar cuáles son las condiciones iniciales del tipo de conocimiento producido, en correspondencia con los requerimientos de los subsistemas sociales. Para Marulanda, López y López (2016), la gestión del conocimiento, representa una forma de agregar o crear valor, a través del uso de herramientas y técnicas específicas, que conduce a optimizar recursos, estimular la creatividad y la innovación.

El análisis plantea la necesidad de una cultura reticular compartida, entre los sectores intervinientes: universidad - gobierno - empresa, en torno al conocimiento como principal agregado de valor de las organizaciones; así como también, la disposición de condiciones de base a su ciclo evolutivo de producción, transferencia y difusión, con el propósito de participar en la generación de innovación y configuración de redes científico - tecnológicas. Consecuentemente, se requieren espacios de red con un tejido relacional de fuertes interacciones, múltiples flujos, donde los actores se integren y converjan, desde sus perfiles reales para la participación, en atención a los grados de formalización de las relaciones establecidas. La red incorpora una estructura formal o informal, constituida por actores territoriales del sector universitario, empresarial y gubernamental, cuya dinámica se fundamenta en flujos relacionales de conocimiento, a través de la disposición de servicios o canales, con el propósito de desarrollar proyectos conjuntos, para satisfacer necesidades y objetivos comunes. Se asume una planeación con horizonte temporal de corto y mediano plazo, a partir de intercambios recurrentes.

\section{Discriminación de actores según perfil de competencias}

Se reconocen los actores que en el ámbito local y regional participan en la configuración de la red científica tecnológica. Las entidades organizacionales generan capacidades para intervenir propositivamente en el referido proceso. Algunos rasgos distintivos de los actores se evidencian en la tabla 2.

Tabla 2: Perfil de los actores participantes de la red

\begin{tabular}{|l|l|l|l|}
\hline$N^{\circ}$ & \multicolumn{1}{|c|}{ Actores } & $\begin{array}{l}\text { Representación } \\
\text { en la red } \\
\text { Conocimiento }\end{array}$ & \multicolumn{1}{c|}{ Participación en la red } \\
\hline 1 & $\begin{array}{l}\text { Unidad de investigación } \\
\text { (LISA - LUZ NPF) }\end{array}$ & $\begin{array}{l}\text { Disponen la estructura de sustento (unidades, procesos y } \\
\text { recursos) para gestionar la producción, transferencia e } \\
\text { intercambio de conocimiento, entre las organizaciones } \\
\text { interactuantes, con base en actividades de investigación, } \\
\text { innovación y desarrollo. }\end{array}$ \\
\hline 3 & $\begin{array}{l}\text { Unidad de investigación } \\
(\text { UnEFA) }\end{array}$ & Conocimiento investigación & Conocimiento \\
\hline 4 & $\begin{array}{l}\text { Unidad de investigación } \\
(\text { UNA) }\end{array}$ & Conocimiento \\
\hline
\end{tabular}


Tabla 2 (continuación)

\begin{tabular}{|c|c|c|c|}
\hline 5 & $\begin{array}{l}\text { Unidad de investigación } \\
\text { (UBV) }\end{array}$ & Conocimiento & \\
\hline 6 & $\begin{array}{l}\text { Gobernación del estado } \\
\text { Falcón - Venezuela } \\
\text { Alcaldía del } \\
\begin{array}{l}\text { Carirubana } \\
\text { Venezuela }\end{array}\end{array}$ & Poder & $\begin{array}{l}\text { Constituido por representantes de organismos del gobierno } \\
\text { local y municipal; participan en el reconocimiento de } \\
\text { necesidades, en cuanto al consumo y aplicación de } \\
\text { conocimiento; generan capacidades para identificar } \\
\text { recursos potenciales y fuentes de financiamiento; definen } \\
\text { estrategias de negociación y concertación entre los actores } \\
\text { locales públicos y privados en el ámbito municipal, para } \\
\text { fortalecer procesos de gestión y participación. }\end{array}$ \\
\hline 7 & $\begin{array}{l}\text { Fundación para el desarrollo } \\
\text { de la ciencia y tecnología. } \\
\text { (FUNDACITE) }\end{array}$ & Conocimiento & $\begin{array}{l}\text { Gestiona la política de ciencia, tecnología e innovación; } \\
\text { define líneas potenciales de investigación como marco de } \\
\text { referencia para derivar planes, programas y proyectos que } \\
\text { respondan a la visión estratégica del Ministerio para la } \\
\text { Ciencia, Tecnología e Industrias Intermedias, en relación } \\
\text { con las necesidades de desarrollo individual y social y } \\
\text { mejoramiento de la calidad de vida. }\end{array}$ \\
\hline 8 & $\begin{array}{l}\text { Cámara de comercio } \\
\text { Cámara de la industria }\end{array}$ & Producción & $\begin{array}{l}\text { Agrupa a los actores dedicados a la actividad comercial en } \\
\text { la zona; definen estrategias para identificar fuentes de } \\
\text { financiamiento externo y asesoría tecnológica, con la } \\
\text { intención de materializar la innovación científica. }\end{array}$ \\
\hline 9 & 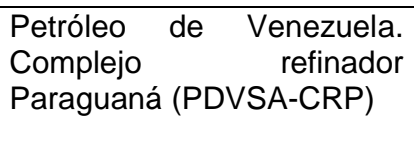 & Producción & $\begin{array}{l}\text { Dentro de la red, es fuente de financiamiento externo, } \\
\text { capacitación técnica y asesoramiento tecnológico con } \\
\text { posibilidades de favorecer las soluciones a problemas de } \\
\text { interés local. }\end{array}$ \\
\hline
\end{tabular}

\section{Concepción filosófica de la red: visión y misión}

Su misión es ser una red de investigación científica - tecnológica para fortalecer los procesos de cooperación y gestión participativa, con sentido de interdependencia, integración y convergencia interdisciplinaria, entre los actores de los diferentes sectores del sistema social, que establecen flujos de relación desde la producción, transferencia y compartición de conocimiento, como base para la innovación tecnológica. Y su visión, se orienta a contribuir a mediano plazo con el desarrollo sostenible de localidades y regiones, a partir de la disposición de una estructura de sustento que viabilice el proceso de producción y transferencia de conocimiento, con fines de generar innovación de base tecnológica.

\section{Exploración de la red con el software UCINET versión 6.5}

Las propiedades estructurales describen la forma en la que los actores se vinculan para formar la red (Streeter y Gillespie, 1992). Este tipo de propiedades se consideran cuando el nivel de análisis es la estructura total de la red. Abordar las propiedades estructurales de la red implica analizar su particular morfología. Al respecto en la figura 1, se visualiza el impacto que fundamenta las relaciones entre los diferentes actores de la red.

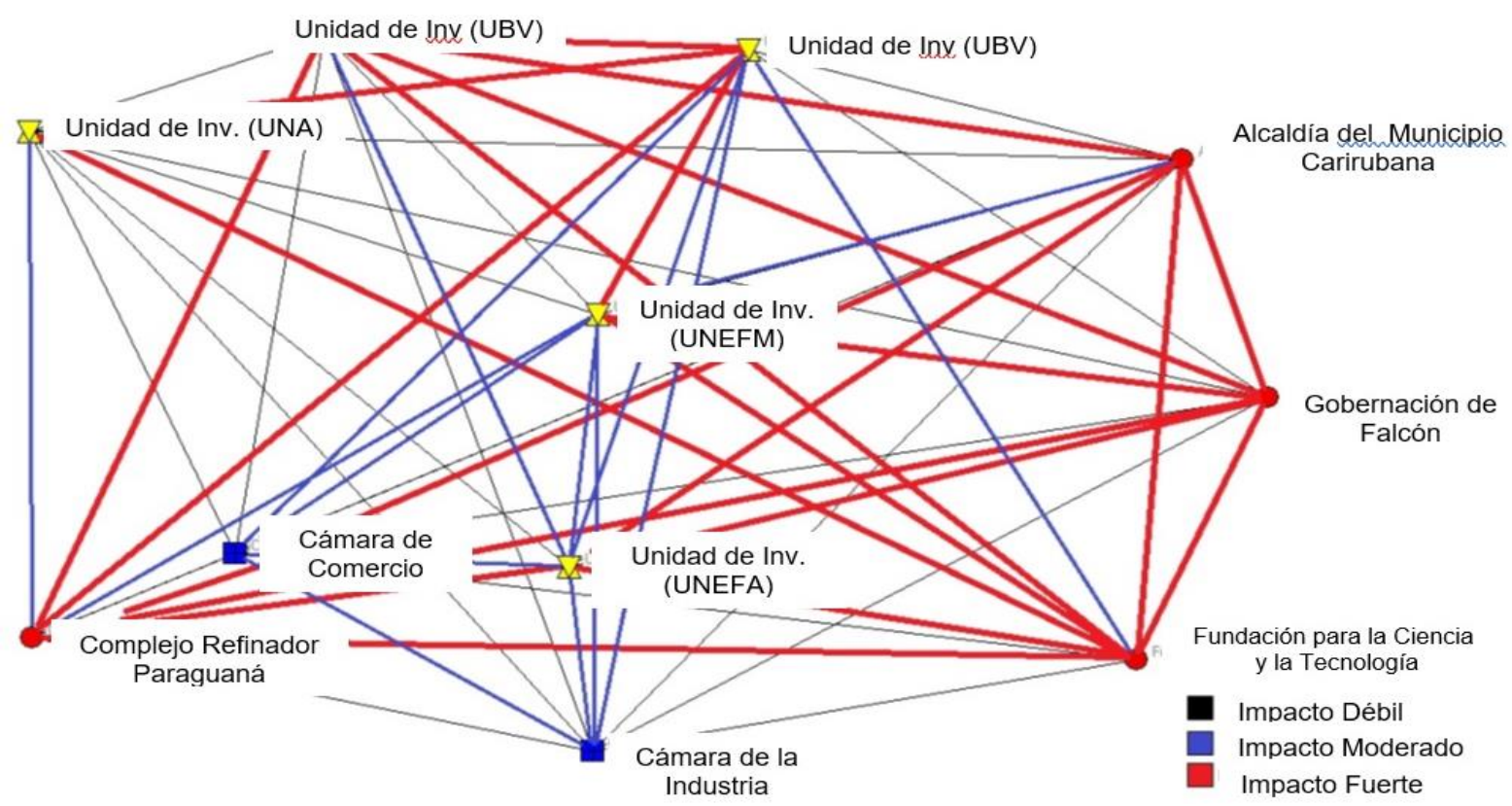

Fig. 1: Impacto de las relaciones entre los actores de la red 
La figura 1 corresponde a una red, con flujos que describen relaciones unidireccionales, de intensidad fuerte, moderada o débil en sus aristas. Interesa conocer su estructura, características en función del perfil de los actores participantes, y cuáles son los nodos centrales. Los círculos, cuadrados y triángulos representan nodos, y cada unión se conforma desde la coincidencia entre uno y otro actor de los actores; en atención a intereses compartidos, se genera una notable densidad de relaciones que se evidencia en la tabla 3.

Tabla 3: Densidad de los Actores

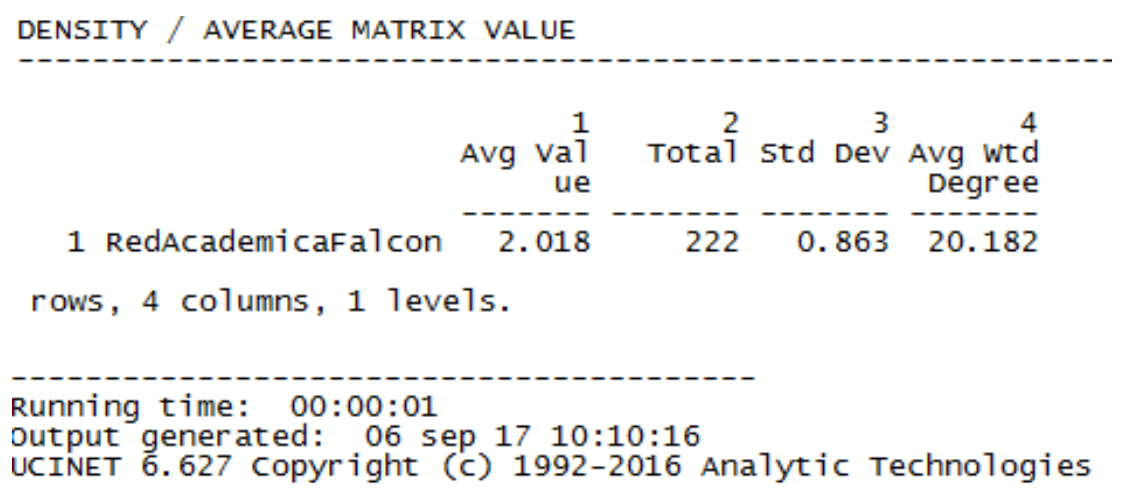

De los valores obtenidos se infiere que en la conformación de la red, se generan importantes grados de conectividad, puesto que alcanza un $201,8 \%$ respecto al potencial de conexión entre los actores. La conectividad es una propiedad uniforme de toda la red, esto se valida con los resultados para la desviación estándar de la densidad (86,3\%), donde se traduce la posibilidad de centralidad en algún nodo; así como también, la conexión entre actores a través de su intervención en el fortalecimiento de procesos de cooperación, para acceder o introducir conocimiento en la red. Para Cabral et al., (2012) y De Rolt, Da Silva y García (2017), las redes se consolidan a partir de la conectividad y dinámica de sus nodos; asocian sus niveles de éxito con la naturaleza de las relaciones interorganizacionales. Se percibe la importancia de generar capacidades de autorregulación, expansión de acciones, fortalecimiento de relaciones horizontales, habilidades interdisciplinarias. En correspondencia a la densidad como propiedad estructural, surgen los grados de centralidad para cada uno de los actores, que se evidencian en la tabla 4.

Tabla 4: Medidas del grado de centralidad para cada uno de los actores

FREEMAN'S DEGREE CENTRALITY MEASURES

\begin{tabular}{|c|c|c|c|c|c|}
\hline & & outDegree & InDegree & NrmoutDeg & $\begin{array}{r}4 \\
\text { NrmInDeg }\end{array}$ \\
\hline $\begin{array}{r}10 \\
6 \\
4 \\
7 \\
1 \\
2 \\
9 \\
5 \\
3 \\
8 \\
11\end{array}$ & $\begin{array}{r}\text { Fundacion para la ciencia y la Tecnologia } \\
\text { Complejo Refinador Paraguana } \\
\text { Unidad de Inv. (UNEFA) } \\
\text { Gobernacion de Falcon } \\
\text { Unidad de Inv. (LISA) } \\
\text { Unidad de Inv. (UNEFM) } \\
\text { Alcadia del Municipio Carirubana } \\
\text { Unidad de Inv. (UBV) } \\
\text { Unidad de Inv. (UNA) } \\
\text { Camara de la Industria } \\
\text { Camara de Comercio }\end{array}$ & $\begin{array}{l}25.000 \\
24.000 \\
23.000 \\
22.000 \\
22.000 \\
21.000 \\
21.000 \\
21.000 \\
15.000 \\
14.000 \\
14.000\end{array}$ & $\begin{array}{l}25.000 \\
24.000 \\
23.000 \\
22.000 \\
22.000 \\
21.000 \\
21.000 \\
21.000 \\
15.000 \\
14.000 \\
14.000\end{array}$ & $\begin{array}{l}83.333 \\
80.000 \\
76.667 \\
73.333 \\
73.333 \\
70.000 \\
70.000 \\
70.000 \\
50.000 \\
46.667 \\
46.667\end{array}$ & $\begin{array}{l}83.333 \\
80.000 \\
76.667 \\
73.333 \\
73.333 \\
70.000 \\
70.000 \\
70.000 \\
50.000 \\
46.667 \\
46.667\end{array}$ \\
\hline
\end{tabular}

DESCRIPTIVE STATISTICS

$\begin{array}{rrrrrr} & & 1 & 2 & 3 & 4 \\ & & \text { OutDegree } & \text { Indegree } & \text { NrmoutDeg } & \text { NrmIndeg } \\ 1 & \text { Mean } & 20.182 & 20.182 & 67.273 & 67.273 \\ 2 & \text { Std Dev } & 3.786 & 3.786 & 12.619 & 12.619 \\ 3 & \text { Sum } & 222.000 & 22.000 & 740.000 & 740.000 \\ 4 & \text { Variance } & 14.331 & 14.331 & 159.229 & 159.229 \\ 5 & \text { SSQ } & 4638.000 & 4638.000 & 51533.336 & 51533.336 \\ 6 & \text { MCSSQ } & 157.636 & 157.636 & 1751.515 & 1751.515 \\ 7 & \text { Euc Norm } & 68.103 & 68.103 & 227.010 & 227.010 \\ 8 & \text { Minimum } & 14.000 & 14.000 & 46.667 & 46.667 \\ 9 & \text { Maximum } & 25.000 & 25.000 & 83.333 & 83.333 \\ 10 & \text { N of obs } & 11.000 & 11.000 & 11.000 & 11.000\end{array}$

Network Centralization (outdegree) $=17.667 \%$

Network Centralization (Indegree) $=17.667 \%$ 
La medida del grado de centralidad o información propia de los resultados, así como los grados de salida y entrada (out -in), normalizados y representados en porcentajes (NrmOut-NrmIn), lleva a identificar el actor central de la red (en función de su influencia al resto), expresados en los grados de entrada; el rasgo permite identificar tres actores con mayor control del conocimiento circulante en la red, a saber: 1) Fundación para el desarrollo de la ciencia y tecnología (FUNDACITE) con 25.000 Grados de entrada Normalizados $83.333 \%$; 2) Petróleos de Venezuela, Complejo refinador Paraguaná (PDVSA-CRP), con 24.000 grados de entrada normalizados $80.000 \%$; 3) Unidad de investigación Universidad Nacional Experimental de las Fuerzas Armadas (UNEFA), con 23.000 grados de entrada normalizados $76.667 \%$. A su vez los mismos actores, se encuentran en las entradas, por tanto reciben un mayor impacto del resto de entidades que dinamizan la red relacional. Son actores con alta motricidad y fuerte interdependencia.

La estadística descriptiva de la desviación estándar (Std Dev) como variación de la media, arroja como resultado 3.786, y el rango promedio de la red (Mean), es de 20.182; por su lado, el grado mínimo y máximo de lazos de los actores en la red, presenta valores que oscilan entre 14 y 25 . Igualmente, se refleja un sum de 222 correspondiente a las relaciones establecidas. De acuerdo a los datos de Ucinet, en promedio los actores, tienen una centralidad en la red bastante aceptable, si se considera que su tamaño es de 11 actores. En cuanto a la desviación estándar se encuentra muy lejos de la media, lo cual indica que existe una baja dispersión. Los grados de centralización de entrada y salida, se reflejan en la ventana de reporte ubicada en la parte inferior de la precitada tabla 2, cuyos resultados son para Network Centralization (Outdegree) $=17.667 \%$ y Network Centralization (Indegree) $=17.667 \%$; estos datos señalan el grado de centralización de la red, ya sea de entrada o salida; proporcionan información acerca de qué tan cerca puede estar una red de comportarse como una red estrella, en donde un solo actor juega un papel central que controla toda la red. Este caso es similar a una red estrella, por el comportamiento del grupo de actores identificados. En relación a este último aspecto, Bravo, Marín y Carrera (2013), destacan la importancia de democratizar acciones colectivas sinérgicas en el espacio de redes; se propicia la creación de una atmósfera de coopetencia entre los actores, para fortalecer su capacidad movilizadora, en atención a las relaciones de poder.

\section{Estrategias participativas para la gestión de la red}

La gestión de la red de cooperación científico - tecnológica en contextos intersectoriales implica diferentes estrategias, cuyas acciones en conjunto actúan como núcleos animadores de las relaciones a potenciar; con el intercambio de conocimiento se promueve la participación concertada de los diferentes actores, mediante procesos de diálogo y en atención a su perfil como agente movilizador del sistema. Se identifican los siguientes planos de intervención:

\section{Formación de los integrantes de la red (estrategia de entrada)}

Es importante identificar participantes del proceso. Su selección obedece a criterios establecidos por los actores centrales, en correspondencia a la concepción, naturaleza y alcance de la red; para visualizar otros actores potenciales, se validan opiniones con base a la retroalimentación cualitativa. Así mismo, es necesario monitorear fortalezas y debilidades, en correspondencia a las habilidades y experticia que demanda el quehacer científico - técnico, propio de la red configurada.

La formación de los actores es uno de los estímulos centrales para la integración de redes de cooperación. Supone la concepción y aplicación de programas tendentes a fortalecer el perfil en investigación, gestión empresarial, tecnológica y de mercado. Dichas estrategias se aplican a través de jornadas de trabajo cuyos propósitos se orienten a: i) Reflexionar acerca de la necesidad de disponer una estructura de sustento para el trabajo en red; ii) Fortalecer actitudes y espacios de confianza para el autoaprendizaje y co - aprendizaje; iii) Definir un marco de trabajo analítico mediante la creación de isomorfismos conceptuales; iv) Identificar fortalezas y brechas a superar en la dinámica funcional de la investigación; iv) Evaluar y validar diseños de investigación; v) Construir conocimiento de base que permita la comprensión de los principios funcionales de la red; v) Disponer de condiciones que permitan el flujo de conocimiento en múltiples direccionalidades; vi) Definir condiciones normativas de funcionamiento. El proceso formativo orienta la dinámica de las redes en correspondencia con las diferentes alianzas estratégicas.

\section{Capacitación profesional de miembros de la red (estrategia de desarrollo)}

La capacitación profesional de los miembros de la red representa una oportunidad para validar prácticas tecnológicas en atención a requerimientos contextuales. Por tal razón se fortalece el perfil de los actores desde el seguimiento a sus expectativas y necesidades de mejoramiento. El propósito se orienta a: i) Compartir vivencias, experiencias, información y conocimiento; ii) Desarrollar experticia y habilidades en el manejo de procesos clave comunes a las organizaciones interactuantes; iii) Fortalecer el perfil de desempeño para la toma de decisiones estratégicas; iv) Aplicar técnicas para el procesamiento de información en la 
gestión de la investigación; v) Definir estrategias de gestión de la red con base a principios de racionalidad, efectividad, eficiencia y eficacia; vi) Propiciar el intercambio con expertos como base para la innovación, transferencia tecnológica y competitividad interorganizacional en la búsqueda de soluciones grupales. vii) Generar estrategias de cooperación para la formación de semilleros de investigación.

\section{Talleres de fortalecimiento de los resultados en red (estrategia de cierre)}

Propician la participación de equipos de investigación en red. Permiten el monitoreo de flujos de información que contribuyen a optimizar y transformar los resultados. Constituyen una estrategia de construcción colectiva, donde se disponen los resultados de las actividades investigativas en función de necesidades y requerimientos; se percibe así la concepción de conocimiento situado y contextualizado (Guzmán, Marín e Inciarte, 2014), con base en la posibilidad de retroalimentar los procesos productivos de las organizaciones interactuantes. La sistematización de experiencias formativas podrá transferirse a situaciones de alcance variado.

Esta estrategia de construcción colectiva fundamenta su dinámica desde una reacción en cadena que permite la concertación, diálogo y negociación, mediante la transacción de datos, información y conocimiento. A través de este proceso se obtendrá: i) Intercambio de ideas, conocimiento y oportunidades; ii) Comprensión de los actores en relación a la conformación de una agenda de investigación flexible, para ajustar los procesos productivos a los requerimientos e intereses individuales y organizacionales; iii) Fortalecimiento de la capacidad de análisis con base en una postura argumentativa o contraargumentativa; iv) Asunción de compromisos formales en cuanto a la confidencialidad de ideas innovadoras. Los integrantes de la red, indistintamente de su rol (coordinador y miembros), organizan reportes de los hallazgos en un informe final, socializado y validado con la participación universidad - gobierno - empresa.

\section{CONCLUSIONES}

Se valida la importancia de la gestión participativa para la integración de redes científico- tecnológicas, cuya dinámica implica flujos de cooperación entre los equipos de trabajo que constituyen nodos de la red; se conceptualizan desde su posibilidad de adaptabilidad a cualquier tipología de red de conocimiento, con similares actores del sector universitario - gubernamental y empresarial, o incluso con la integración de instituciones de otra naturaleza. El análisis de redes concibe soluciones en correspondencia con las condiciones que disponen los sectores intervinientes, en atención a su perfil científico - tecnológico, para fortalecer relaciones de cooperación.

La configuración supone la sustentabilidad de vías de conectividad, para el acercamiento progresivo de los actores; se acortan distancias, minimizan incompatibilidades y precisan alianzas de conocimiento, con respaldo en las capacidades y fortalezas propias de los sistemas interactuantes, que comparten objetivos para la búsqueda de fines comunes. Del análisis obtenido, se ha pretendido solo un abordaje de la red en atención a categorías clave, como: relaciones y estructura, densidad, características de los actores e identificación de los nodos centrales. Este estudio derivó la existencia de flujos de densidad variable, con base en el intercambio de ciencia y tecnología que tienen lugar a través de las redes de cooperación.

\section{REFERENCIAS}

Azua, J. y A. Andersen, Alianza Competitiva para la Nueva Economía, $1^{\text {a }}$ Ed., 60-85, Mcgraw-Hill, Madrid, España (2000)

Borgatti, S., Centrality and Network Flow, Social Networks, 27(1), 55-71(2005)

Borgatti, S., M. Everett y L. Freeman, Ucinet 6 for Windows: Software for Social Network Analysis, Harvard, MA: Analytic Technologies (2002)

Bozdogan, H. y O. Akbilgic, Social Network Analysis of Scientific Collaborations across Different Subject Fields, doi: 10.3233/ISU-130715, Information Services \& Use, 33(3), 219-233 (2013)

Bravo, O., F. Marín-González y M. Carrera, Redes Inter-organizacionales y Desarrollo Local, Opción, 29(70), 86-103 (2013)

Cabral Flecha, A., A. Castro, J. Alves y A. Tristao, Redes de Empresas e seus Efeitos sobre o Turismo, RAE-Revista de Administração de Empresas, 52(4), 386 - 406 (2012)

Cárdenas, M., T. Rivas, L. Ramírez y N. Simón, Análisis de la Estructura de una Red de Conocimiento en México, Revista de Ciencias Sociales, XXI(4), 521 - 537 (2015)

Cardozo - Montilla, M., Evolución de las Redes de Investigación en el Contexto Universitario Latinoamericano: Análisis Comparativo del Impacto de los Esquemas de Investigación de Colombia y Venezuela, Revista Venezolana de Análisis de Coyuntura, XVIII (2), 149-162 (2012) 
Cunill-Grau, N., La Intersectorialidad en las Nuevas Políticas Sociales: Un Acercamiento Analítico-Conceptual, Gestión y Política Pública, 23(1), 5-46 (2014)

De Rolt, C., D. Da Silva y F. García, Network Analysis as a Management Tool for Inter-organizational Projects, Gestão \& Produção, 24(2), 266-278 (2017)

Dushnitsky, G. y J. Miles, Limitations to Interorganizational Knowledge Acquisition: The Paradox of Corporate Venture Capital, Strategic Management Journal, 30(10), 1045-1064 (2009)

Dyer, J. y H. Singh, The Relational View: Cooperative Strategy end Sources of Interorganizational Competitive Advantage, Academy of Management Review, 23 (4), 660-679 (1998)

Espinoza, R., Redes de Investigación y Desarrollo, Estructuras Organizacionales para la Transferencia de Conocimiento, Multiciencias, 11(3), 235-243 (2011)

Etzkowitz, H., Innovation in Innovation: The Triple Helix of University-Industry-Government Relations, Social Science Information, 42(3), 293-337 (2003)

García, A., H. Díaz y J. Arias-Pérez, Capacidades de Tecnologías de Información y Capacidades de Negocio Electrónico (E-Business): Efecto Mediador de la Capacidad de Absorción, doi: 10.4067/S0718-07642017000100006, Inf. Tecnol. 28(1), 47-64 (2017)

Gutiérrez, C., M. Carrera y otros tres autores, Integración de Redes Académicas para la Gestión del Desarrollo Endógeno Regional, Multiciencias, 6(3), 257-263 (2006)

Gutiérrez, L., Sistema Teórico Explicativo sobre la Dinámica de las Redes Interorganizacionales, Revista Venezolana de Gerencia, 22(77), 97-120 (2017)

Guzmán, I., R. Marín y A. Inciarte, Innovar para Transformar la Docencia Universitaria. Un modelo para la Formación por Competencias, $1^{\text {a }}$ Ed., 30 - 50, Astro Data SA, Maracaibo, Venezuela (2014)

Huang, K., X. Chen y otros tres autores, Heterogeneous Cooperative Belief for Social Dilemma in Multi-agent System, Applied Mathematics and Computation, 320(C), 572-579 (2018)

Krichel, T. y N. Bakkalbasi, Social Network Analysis of Research Collaboration in the EconoMics Community, Journal of Information Management and Scientometrics, 43(1), 1-15 (2006)

Li, L., F. Catalá-López y otros ocho autores, The Global Research Collaboration of Network Meta-Analysis: a Social Network Analysis, PloS One, 11(9), 1-15 (2016)

Lovera, M., E. Castro y otros tres autores, Evolucionismo Económico desde la Perspectiva de Nelson y Winter, Multiciencias, 8, 48-54 (2008)

Marín-González, F., M. Riquett y otros tres autores, Gestión Participativa y Calidad Educativa en el Contexto del Plan de Mejoramiento Institucional en Escuelas Colombianas, Opción, 33(82), 344-365 (2017)

Marín-González, F., L. Cabas., L. Cabas y A. Paredes-Chacín, Formación Integral en Profesionales de la Ingeniería. Análisis en el Plano de la Calidad Educativa, doi: 10.4067/S0718-50062018000100013, Formación Universitaria, 11(1), 13-24 (2018)

Marulanda, C., M. López y F. López, La Cultura Organizacional y las Competencias para la Gestión del Conocimiento en las Pequeñas y Medianas Empresas (PYMEs) de Colombia, doi: 10.4067/S0718-07642017000100006, Inf. Tecnol., 27(6), 3-10 (2016)

Obando, L. y J. Delgado, Diseño de una Red de Participación de Actores para la Gestión de los Sistemas de Financiamiento, Visión Gerencial, 6 (2), 298-315 (2007)

Osca-Lluch, J. y F. González-Sala, Scientific Networks and Research Groups Development. Educational Psychology case in Spain during the Five-Year Periods 2004-2008 and 2009-2013, Anales de Psicología / Annals of Psychology, 33(2), 356-364 (2017)

Powell, W., Neither Market nor Hierarchy Network Forms of Organization, Research on Organizational Behavior, 12, 295$336(1990)$

Robinson- García, N., R. Rodríguez- Sánchez y otros tres autores, Análisis de Redes de las Universidades Españolas de acuerdo a su Perfil de Publicación en Revistas por Áreas Científicas, Revista Española de Documentación Científica, 36 (4) $20-27$ (2013)

Sacchetti, S. y E. Tortia, The Extended Governance of Cooperative Firms: Inter-Firm Coordination and Consistency of Values, Annals of Public and Cooperative Economics, 87(1), 93-116 (2016)

Sebastián, J., Las Redes de Cooperación como Modelo Organizativo y Funcional para la I+D, Redes, 7(015), 97-111 (2000)

Simanca, M., L. Montoya y C.Bernal, Gestión del Conocimiento en Cadenas Productivas: El Caso de la Cadena Láctea en Colombia, doi: 10.4067/S0718-07642017000100006, Inf. Tecnol., 27(3), 93-106 (2016)

Smith, P. y A. Van de Ven, Developmental Processes of Cooperative Interorganizational Relationships, The Academy of Management Review, 19(1), 90-118 (1994)

Streeter, C. y D. Gillespie, Social Network Analysis, Journal of Social Service Research, 16 (1/2), $201-222$ (1992) 
Uzzi, B, Social Structure and Competition in Interfirm Networks: The Paradox of Embeddedness. Administrative Science Quarterly, 42(1), 35-67(1997)

Vega, M. e Y. Rojo, Contribución al Desarrollo Local de la Conformación de Redes Intersectoriales, Opción, 26 (63), 105$116(2010)$

Villanueva-Felez, A., A. Fernández-Zubieta y D. Palomares-Montero, Propiedades Relacionales de las Redes de Colaboración y Generación de Conocimiento Científico: ¿Una Cuestión de Tamaño o Equilibrio?, Revista Española de Documentación Científica, 37(4), 068, (2014)

Wasserman, S. y K. Faust, Social Network Analysis: Methods and Applications, Vol. 8, Cambridge University Press, Cambridge, Inglaterra (1994)

Weber, C., B. Bauke y V. Raibulet, An Empirical Test of the Relational View in the Context of Corporate Venture Capital, Strategic Entrepreneurship Journal, 10(3), 274-299 (2016)

Weber, C., K. Weidner, A. Kroeger y J. Wallace, Social Value Creation in Inter-Organizational Collaborations in the Notfor-Profit Sector-Give and Take from a Dyadic Perspective, Journal of Management Studies, 54(6), 929-956 (2017) 